\title{
Arabidopsis Sucrose Transporter 4 (AtSUC4) is Involved in Root Response of High Content Sucrose-Growth Via IAA- and ABA-Dependent Manner
}

\section{Si-Wen Liu}

South China Agricultural University

Li-Ding Zhang

South China Agricultural University

Jia-Yu Gao

South China Agricultural University

Tian-Tian Dong

South China Agricultural University

Tong Zhang

South China Agricultural University

Jian-Mei Long

South China Agricultural University

Chang-Cao Peng ( $\nabla$ ccpeng@scau.edu.cn )

South China Agricultural University

\section{Research Article}

Keywords: AtSUC4, root response, sucrose-growth, auxin, abscisic acid

Posted Date: January 22nd, 2021

DOI: https://doi.org/10.21203/rs.3.rs-148254/v1

License: (c) (i) This work is licensed under a Creative Commons Attribution 4.0 International License. Read Full License 


\section{Abstract}

Source-to-sink transport of sucrose mediated by sucrose transporters (SUCs) is one of the major determinants of plant growth. However, the role of AtSUC4, the only member of Group IV sucrose transporter in Arabidopsis, was undervalued in sink organ during seedling period. In our study, the primary root length of the atsuc4 mutants was significantly longer than that of the wild-type (WT) under exogenous $4 \%$ and $6 \%$ sucrose treatment. But this phenotype could not be imitated by external application of glucose or mannitol. It means that the atsuc4 mutants were insensitive to high sucrose stress compared with WT. Meanwhile, HPLC-MS/MS results showed that the root of atsuc4 mutants accumulated less sucrose and ABA and more IAA content compared with WT on $4 \%$ and $6 \%$ sucrose supplementation. Transcriptome analysis showed that many key genes involved in IAA and ABA signals were respective stimulated and repressed in the atsuc4 mutants, respectively. Taken together, we concluded that the deficiency of AtSUC4, not only reducing the transported and uptaked of sucrose, but also as a signal, may be collaborated with IAA and ABA to regulate root growth under high sucrose stress. This study confirmed the new function of AtSUC4, and provided an promising candidate gene for improving tolerance to high sucrose stress.

\section{Introduction}

In most plants, sucrose plays an important role in stress tolerance, serving as an osmolyte, a signaling molecule and/or a nutritional substance [1]. Most sucrose is derived from photosynthetic source organ leaves, and transported into sink organs, including flowers, stems, roots and seeds. This way of sucrose exchange requires transport proteins to mediate sucrose uptake or release from cells or subcellular compartments. Thus, not surprisingly, sucrose transporters (SUCs or SUTs) are critical for plant growth and development [2]. Sucrose synthesized in the source cells is exported to the apoplasm, probably by the SWEET efflux proteins, then imported into the sieve elements and companion cells complexes (SE-CCCs) via sucrose transporters (SUT or SUC) in most plants [3]. SUCs are $\mathrm{H}^{+} /$sucrose symporters that utilize the proton motive force (PMF) present across the plasma membrane (PM) of the SE-CCCs to load sucrose against its concentration gradient into the phloem [4]. Multiple SUT genes have been identified and initially falled into three subgroups (SUT1, SUT2 and SUT4 subfamily) but later into four distinct groups (Group I, II, III and IV) according to phylogenetic analysis [5, 6]. Among them, most members of the Group II are high-affinity/low-capacity sucrose transporters with $\mathrm{Km}$ values ranging between $139 \mu \mathrm{M}$ and $1.5 \mathrm{mM}$ and described as plasma membrane-localized transporters, whereas members of the Group IV (with the exception of OsSUT2 from rice) are low-affinity/high-capacity sucrose transporters with $\mathrm{Km}$ values ranging from $5 \mathrm{mM}$ to $6 \mathrm{mM}$ and suggested as the tonoplast-localized transporters [7]. There are 9 SUCs in Arabidopsis (Arabidopsis thaliana), among which 7 members (AtSUC1, 2, 5-9) belong to Group II, but only AtSUC1 and AtSUC2 were well studied due to their high expression levels [4]. AtSUC1 could control dehiscence of anthers and pollen tube growth and AtSUC2 was responsible for phloem loading [8, 9]. SUTs in group IV showed distinct functions in different plant species indicating a versatile role of the members in this group involving plant growth and development $[7,10]$. AtSUC4, the only member of 
Group IV sucrose transporter in Arabidopsis, can also catalyze the transport of sucrose from the vacuolar lumen into the cytoplasm in the cells [10]. Due to the low expression level of AtSUC4, the aerial part of atsuc4.1 (cs856419) mutant behaved essentially as the WT in sucrose content, germination, development and response to cold treatment when cotyledons were fully unfolded. The AtSUC4-overexpressing lines showed a 30\% reduction in sucrose content in aerial parts compared with WT under $3 \%$ sucrose treatment [11]. However, little is known about the role of AtSUC4 in the root, a typical sink organ that relies exclusively on an source leaves and external import of sucrose, especially in the high sucrose surrounding.

In Arabidopsis, primary root elongation rate was basically dependent on sugar concentration in the medium, and a good correlation between the elongation rate and sugar content in primary roots was found [12]. In addition, sugars transported into the root also transmit as signals. In young developing seedling root of Arabidopsis, Kircher and Schopfer demonstrated that sucrose transported into root acts as a necessary and sufficient signal regulating root elongation together with light [13]. The expression patterns of AtSUC1 and AtSUC2 displayed contrasting roles during the night, showing increased transcript accumulation of AtSUC2 (sucrose loading in phloem) in leaves and AtSUC1 (sucrose unloading) in roots $[14,15]$. However, the function of AtSUC4 in root development was uncertain, and a clear linking on the interaction between sucrose transport and root growth remains to be elucidated.

In addition to being directly regulated by sucrose, root development also results from the concerted action of multiple plant hormones. Among these, auxin stands out as a key instructive signal on which many other plant hormones converge to regulate root development [16]. Moreover, exogenous sucrose could promote the root growth through sugar hormone crosstalk in Arabidopsis and peach [17, 18]. Further, it was found that exogenous sucrose increased the expression of auxin synthesis- and transport-related genes in roots, resulting in auxin accumulation in the root system [18]. Besides, ABA signaling also plays a critical role in regulating root growth and root system architecture [19]. Yu et al revealed that the OSERF2 was required for the root architecture and ABA-response by regulating the expression of some genes involved in sugar metabolism and hormone signaling pathways [20]. Additionaly, Yuan et al reported that excessive exogenous glucose inhibits primary root growth via $A B I 5$, which represses PIN1 accumulation and auxin activity in Arabidopsis [21]. To date, however, the molecular mechanism of crosstalk among sucrose transporters, IAA and $A B A$ regulating root growth under stress conditions remains unclear.

To gain more information about sucrose transport regulation in root with emphasis on AtSUC4, expression patterns and subcellular localization of AtSUC4 were performed firstly. Atsuc4 mutant lines were generated and showed longer root length under high exgeonous sucrose stress. Comparision of sugar and hormone (IAA and ABA) content in roots between WT and mutant lines were analyzed.

Transcriptome were carried out, and indicated that expression of several sucrose transporters, IAA- and ABA-related genes were changed in mutant lines. This study confirmed the new function of AtSUC4, and provided insights into the regulation of AtSUC4 in root growth under high sucrose stress. 


\section{Materials And Methods}

\section{Strains materials and growth conditions}

Arabidopsis thaliana (ecotype Col-0) and atsuc4 mutants were grown in growth chamber in soil under long-day (LD) conditions ( $16 \mathrm{~h}$ light / $8 \mathrm{~h}$ dark) at $22^{\circ} \mathrm{C}$ and $70 \%$ relative humidity, light was present at 100-120 $\mu \mathrm{mol} . \mathrm{m}^{-2} . \mathrm{s}^{-1}$. Before germination, plates were incubated at $4{ }^{\circ} \mathrm{C}$ for $2 \mathrm{~d}$ in the dark and subsequently transferred to the growth chamber. Escherichia coli strain DH5a was used for all cloning steps. Transformation of Arabidopsis was performed using Agrobacterium tumefaciens strain GV3101 [22].

\section{Generation of Arabidopsisatsuc4 mutant lines}

Three types of atsuc4 mutant lines were used in this study. The atsuc4 mutant (cs93419) was obtained from the Nottingham Stock Centre (http://arabidopsis.info/), and screened the AtSUC4 point mutation homozygous with no ERECTA gene mutation, which was named as suc4-1. To construct AtSUC4 single gene knockout vectors contained two target sites (TCACAGAGTCACTCGCAACC and CTCGTTGGGCATAGTAGCGA), we adopted the CRISPR/Cas9 system created by Ma et al [23]. The homozygous transformed plants with vector-free were generated, which was called suc4-2. The AtSUC4 TDNA insertion line named suc4-3 (WiscDsLox450E10) was obtained from the Nottingham Stock Centre (http://arabidopsis.info/). The position of the T-DNA insertion was determined by sequencing a PCR product obtained from suc4-3 genomic DNA [11]. For the AtSUC4 complement-expressors (marked as AtSUC4/ suc4-1), the AtSUC4 promoter and gDNA sequence fragments were amplified from the total DNA of leaves, and inserted into the BamH I- and EcoR I-digested plant transformation vector PCAMBIA1300 [24] by ClonExpress MultiS One Step Cloning Kit (Vazyme). The resulting plasmid pCAMBIA1300pSUC4::gSUC4-GFP was used for suc4-1 mutant transformation. Primer pairs for vector construction and identification of atsuc4 mutants were shown in Data S1.

\section{Subcellular localization assay of AtSUC4}

Subcellular localization of AtSUC4 was detected by four methods. For the method of Arabidopsis stable transformation system, the promoter (998 bp upstream of ATG) and CDS sequence were amplified, and cloned into pCAMBIA1301 vector with Citrine fluorescent protein coding sequence, which was kindly provided by Dr. Erik Nilesen (University of Michigan). As a result, the construct pCAMBIA1301pSUC4::.cSUC4-Citrine was generated, and subsequently transformed into WT plants. Imagies were obtained using the roots of transgenic homozygous lines. The second method of transient expression system of Nicotiana benthamiana leaves was used. In this method, the plasma membrane marker tag AtCERK1-DsRed [25] and tonoplast marker tag AtCBL6-OFP plasmids [26] were used as positive controls, which were provided by Dr. Xian'an Xie (South China Agricultural University). GV3101 were transformed with plasmid pCAMBIA1300-pSUC4::gSUC4-GFP described above and each positive marker respectively, followed by co-infiltration into Nicotiana benthamiana leaves with a final $\mathrm{OD}_{600}=0.5-0.7$. Plants were incubated for 36-48 $\mathrm{h}$ before imaging. We used the transient expression system of Arabidopsis protoplast 
as the third method to check the subcellular localization of AtSUC4. Protoplast isolation and transformation of rosette leaf were performed according to Robert et al [27]. The vector pEarley10135S:::CAtSUC4-GFP was generated and used for protoplast transient transformation. All the fluorescence were detected using a Leica TCS SP5 confocal microscope. The excitation wavelengths used to detect fluorescences were $488 \mathrm{~nm}$ for GFP, $512 \mathrm{~nm}$ for Citrine, and $543 \mathrm{~nm}$ for DsRed and OFP, while the emitted fluorescences were collected from 500 to $550 \mathrm{~nm}$ for GFP, $519 \mathrm{~nm}$ to $650 \mathrm{~nm}$ for Citrine and 565 to 615 $\mathrm{nm}$ for DsRed and OFP. The fourth method was immunegold labeling of AtSUC4 which essentially performed as described previously [28] using the mouse anti-AtSUC4 monoclonal antibody (Xi Qin, unpublished).

\section{Verification of expression pattern of AtSUC4 at tissue level}

To verify expression pattern of AtSUC4 during Arabidopsis development at tissue level, we created the pAtSUC4:: GUS transgenic plants under the control of AtSUC4 promoter. A 998-bp fragment before coding region of AtSUC4 was amplified from total DNA of leaf, subcloned into the entry vector pDONR207 and inserted upstream of the GUS into destination vector pHGWFS.7 [29], yielding construct pAtSUC4::GUS, which was used for Agrobacterium tumefaciens transformation. Primer pairs used were shown in Data S1. Samples including the whole seedlings (grown for $4 d, 14 d$ and $25 d$ period), flowers (grown for $35 d$ period), siliques and seeds (grown for $50 \mathrm{~d}$ period) were stained according to standard protocols [11]. For each sample, we analyzed 10 to 15 independent lines, and representative results were presented. Photograph were captured by stereomicroscope (Leica MZFL III; Leica Microsystems, Bensheim, Germany).

\section{Genes expression level detection}

For gene expression analysis, total RNA was isolated from roots of different plants using the Plant RNA Extraction Kit (Omega, Norcross, GA, USA), and cDNA was synthesized using HiScript II 1st Strand cDNA Synthesis Kit (+gDNA wiper) (Vazyme). Reverse transcription-quantitative real time PCR (RT-qPCR) was conducted using FastStart DNA Master SYBR Green I (Roche) on the Roche LightCycler 480 System (Roche Applied Science), with ACTIN2 as internal control [30]. Three independent biological replicates and four technical replicates were performed. The primer sequences were listed in Data S1.

\section{Measurement of root length and seed germination rate between WT and mutant lines}

For sugars tolerance, the seeds of WT and atsuc4 mutant lines were sown on MS solid media containing different concentrations of sucrose, glucose and mannitol after sterilizing the seeds $(0 \%, 2 \%, 4 \%$ and $6 \%)$. Bright-field images of different plants were captured at $6 \mathrm{~d}$ after sowing using Canon $60 \mathrm{D}$ camera. Root length was measured by Image $\mathrm{J}$ software. For the phenotype comparision between WT and mutants, plants were grown in soil under LD condition. The number of rosette leaves was recorded at $25 \mathrm{~d}$, and the plant height was measured at $50 \mathrm{~d}$. Three biological replicate and more than 30 plants for each replicates were used for analysis. For seed germination rate, the WT and mutant seeds were sown on MS solid media containing different concentrations of sucrose $(0 \%, 2 \%, 4 \%$ and $6 \%)$ and seed germination were 
calculated after 24 h, 48 h, 60 h and 72 h. Each treatment was performed with three biological replicates, at least comprising 100 seeds in each experiment.

\section{Analysis of carbohydrate content}

Preparation of plant materials and determination of carbohydrate content via ion chromatography was performed as previous described [30]. WT, atsuc4 mutants and AtSUC4/ suc4-1 seeds were sown on MS solid media containing different concentrations of sucrose $(0 \%, 2 \%, 4 \%$ and $6 \%)$. Roots were collected and quickly grinded in liquid nitrogen. An aliquot of each individual sample was precisely weighed (20 $\mathrm{mg}$ ) and transferred to an eppendorf tube. After adding the addition of $500 \mu \mathrm{L}$ of extract solvent (ethanolwater, 8:1) and including the isotope as internal stand with the fix concentration. The samples were vortexed sonicated. The homogenate and sonicate circle were repeated for 3 times, followed by incubation at $90{ }^{\circ} \mathrm{C}$ for $30 \mathrm{~min}$ and centrifugation at $12000 \mathrm{rpm}$ for $15 \mathrm{~min}$. The supernatant was transferred to an auto-sampler vial for UHPLC-MS/MS analysis to measure glucose and fructose. Another aliquot was further diluted for 10 times for UPLC-MS/MS analysis to quantitate the sucrose. When the concentrations of metabolites were beyond the standard curve range, the samples were diluted accordingly to place their concentrations within the range. The final concentration in $\mu \mathrm{g} / \mathrm{mg}$. FW equals the calculated concentration multiplied by the dilution factor.

\section{The determination of IAA and ABA content in roots}

Roots were collected from WT and suc4-1 mutant grown on MS solid media with $2 \%$ and $6 \%$ sucrose supplementation under LD condition at $6 \mathrm{~d}$ after sowing, with three biological replicates (approximately $0.5 \mathrm{~g}$ fresh weight per sample). And the contents of endogenous IAA and ABA were determined according to a previously reported method with modification [31]. IAA and ABA analysis were performed on a Thermo Scientific Ultimate 3000 UHPLC System equipped with a Thermo Scientific TSQ Quantiva-Stage Quandrupole Mass Spectrometer (http://www.greenswordcreation.com).

\section{RNA sequencing and sequence analysis}

Total RNA was isolated from the 6 d-old seedling roots of WT, suc4-1 and suc4-2 plants which grown on MS solid media containing different concentrations of sucrose $(2 \%, 4 \%$ and $6 \%)$ in three biological replicates. RNA samples were treated with DNase before being quality checked using an Agilent 2100 Bioanalyzer. Total RNA was prepared for a strand-specific TruSeq ${ }^{\text {TM }}$ RNA-seq library, and all 27 samples were sequenced on an Illumina HiSeq 4000, with 150 bp paired end reads. Differential expression analysis was performed using the DEGSeq $\mathrm{R}$ package (1.12.0). $P$-values were adjusted using the Benjamini \& Hochberg method. A corrected $P$-value of 0.05 and $\log _{2}$ (Fold Change) of 1 were set as the threshold for significantly differential expression. Gene ontology enrichment analysis of differentially expressed genes was implemented by the GOseq R package, in which gene length bias was corrected. Gene ontology terms with a corrected $P$-value less than 0.05 were considered significantly enriched by differentially expressed genes. All sequences generated in this study have been deposited in the National 
Center for Biotechnology Information Sequence Read Archive (https://www.ncbi.nlm.nih.gov/sra/) with project number PRJNA666009.

\section{Statistical analyses}

The overall data were statistically analyzed using SPSS 20 software (IBM China Company Ltd., Beijing, China). One-way or two-way ANOVA with Duncan test at the $5 \%$ level was used to test differences between multiple samples. Data were presented as means and SEs using Microsoft Excel.

\section{Results}

\section{Expression pattern and subcellular localization of AtSUC4}

To reveal the spatio-temporal expression of AtSUC4 in Arabidopsis, we generated a construct (pAtSUC4::GUS) harboring the GUS reporter gene driven by the endogenous AtSUC4 promoter and transformed into Col-0. As shown in Figure 1A-B, AtSUC4 was mainly expressed in vasculature cells of leaves and mature portion of $4 \mathrm{~d}$ and $14 \mathrm{~d}$-old seedling roots. In $25 \mathrm{~d}$-old plants, GUS staining covered the entire root and juvenile leaves but not mature leaves (rosette) (Fig. 1C). In the inflorescence, petals, styles and the developing flower buds showed the presence of GUS staining (Fig. 1D). Specifically, we detected the GUS activity in vasculature of petals and styles in mature flower (Fig. 1E). In addition, Figure $1 \mathrm{~F}$ showed that strong GUS activity was detected in vascular tissues of siliques at 1 DAF (days after flowering) or 6 DAF and embryo at 15 DAF. To investigate whether the AtSUC4 expression was induced by sucrose, pAtSUC4::GUS seedlings were grown on the medium with different concentrations of sucrose $(0 \%, 2 \%, 4 \%$ and $6 \%)$ for $7 \mathrm{~d}$ and then stained for GUS activity detection. As shown in Figure $2 \mathrm{~A}$, with the increase of sucrose concentration in the medium, the activity of GUS became gradually higher, suggesting that AtSUC4 expression was significantly induced by sucrose. This result were further surpported by GUS expression analysis using RT-qPCR (Fig. 2B).

To verify the subcellular localization of AtSUC4, the construct encoding AtSUC4-GFP under endogenous promoter was generated and co-expressed with plasma membrane marker or tonoplast marker in $\mathrm{N}$. benthamiana leaves by infiltration with Agrobacteria tumefaciens. The result demonstrated that AtSUC4 was co-located with both plasma membrane and tonoplast marker, indicating that AtSUC4 was localized to plasma membrane and tonoplast, but compared to the plasma membrane labelling, the signal in tonoplast was quite faint (Supplementary Fig. S1). We further checked the result with the AtSUC4-Citrine fusions protein expressed in the root of Arabidopsis. However, AtSUC4-Citrine gave clear and strong labelling of the plasma membrane but not tonoplast (Fig. 3A). Immune colloidal gold assay and transient transformation of Arabidopsis protoplasts with AtSUC4-GFP vector showed that AtSUC4 was localized to plasma membrane (Fig. 3B-C).

\section{Atsuc4 mutants exhibited longer roots under high sucrose stress}


In order to observe the effect of AtSUC4 mutation on plant growth, except the suc4-3 (T-DNA insertion from Nottingham Stock Centre), we generated suc4-1 by EMS mutagenesis and suc4-2 by CRISPR/Cas 9 mutagenesis (Fig. 4A). The suc4-1 point mutation (C502A) and suc4-2 inserted mutation (A was inserted between nucleotides 29 and 30) introduced stop codon (TAA), resulting an early termination of AtSUC4 protein translation (Fig. 4B). The AtSUC4 transcript in all the three mutants were significantly lower than WT, whereas it was similar to that of the WT in the AtSUC4/suc4-1 (Fig. 4C-D). Under the LD conditions, both plant height and leaf number were similar in all genotypes (Fig. 4E-F, Supplementary Fig. S2A-B). The root length of WT was similar to all the three atsuc4 mutants on medium supplemented with light sucrose concentration, including $0 \%, 0.5 \%, 1 \%, 1.5 \%$ or $2 \%$ sucrose (Supplementary Fig. S2C). Although the maturation of the embryo in suc4-1 was 1 day later than WT (Supplementary Fig. S2D), it did not affected the normal development of the embryo. These data demonstrated that there was no difference in phenotype between WT and atsuc4 mutants plants on the sucrose-free or light concentration of sucrose condition.

To test whether phenotypes of atsuc4 mutants were altered in response to high content sucrose, we calculated the primary root length and germination rate of atsuc4 mutants and WT under different concentrations of sugars $(0 \%, 2 \%, 4 \%$ and $6 \%)$. The results showed that as the sucrose concentration became higher, the root length in all lines were gradually decreased (Fig. 5A-B), indicating that high concentration of sucrose would inhibit the root growth. Interestingly, the primary root of the atsuc4 mutants were significantly longer than that of the WT and AtSUC4/ suc4-1 when the sucrose concentrations in the medium were raised to $4 \%$ and $6 \%$ (Fig. 5A-B). Besides, we explored whether the high concentration glucose and/or osmotic stress would cause the difference of primary root length among WT, atsuc4 mutants and AtSUC4/ suc4-1 by external application of a gradient concentration glucose and mannitol (simulated osmotic stress). As shown in Supplementary Figure S3 and S4, no differences were found in the root length among these three genotypes of plants under the same treatment. Besides, we found that the germination rate of suc4-1 was similar to WT on agar medium supplemented with $0 \%, 2 \%$ and $4 \%$ sucrose, respectively (Supplementary Fig. S5). However, the germination of suc4-1 seeds was 1 to 2 days later than WT when the concentration of sucrose was $6 \%$. Taken together, these results suggested that disruption of AtSUC4 in atsuc4 mutants repressed the inhibition of high concentration sucrose on root growth, but not on germination rate. The deficiency of AtSUC4 might be important for tolerance of high sucrose stress.

\section{Atsuc4 mutants accumulated less sucrose and ABA and more IAA than WT in roots under high sucrose stress}

To investigated whether the change in root length was caused by the sucrose content in atsuc4 mutants, we then compared the main sugars (including sucrose, glucose and fructose) content in roots of above plants. The results revealed that an obviously reduced sucrose content in root was shown in atsuc4 mutants compared with WT and AtSUC4/ suc4-1 on medium with $2 \%, 4 \%$ and $6 \%$ sucrose supplementation (Fig. 6A), suggesting negatively correlation with the length of roots. To a lesser extent, the glucose content of suc4-1 and suc4-3 roots were decreased compared with WT on medium 
supplemented with $4 \%$ and $6 \%$ sucrose, whereas no significantly difference between suc4-2 and WT (Fig. 6B). The content of fructose in three atsuc4 mutants were significantly lower than that of WT and AtSUC4/ suc4-1 on medium without sucrose. As the concentration of sucrose increases to $6 \%$, markedly reduced fructose content was detected in suc4-2 compared with WT, but no significantly differences in WT, suc4-1 and suc4-3 (Fig. 6C). These results showed that atsuc4 mutants exhibited longer root length compared with WT due to a less sucrose accumulation during high sucrose stress.

In order to verify whether the reduction of the root length of the atsuc4 mutants crossed with the IAA and ABA signal pathway under high sucrose stress, we detected the IAA and ABA contents in WT and suc4-1 roots (Tab. 1). The result showed that the content of IAA in suc4-1 and WT was decreased in the $6 \%$ sucrose supplementation compared with $2 \%$ sucrose, however, it was significantly higher in suc4- 1 than WT, suggesting that the less reduction of IAA might alleviate the inhibition of root growth under high sucrose stress. On the contrary, $6 \%$ sucrose stimulated the accumulation of ABA in both suc4-1 and WT, and less ABA was accumulated in the suc4-1 compared with WT.

\section{Analysis of global gene expression change in atsuc4 mutants under different sucrose condition}

To better understand the role of AtSUC4 at the molecular level under high sucrose stress, comparative transcriptome analysis was performed between two atsuc4 mutants (suc4-1 and suc4-2) and WT using root which grown $6 \mathrm{~d}$ under $2 \%, 4 \%$ and $6 \%$ sucrose treatments. A total of 1,259 (581 up, 678 down) and 3,191 (1,474 up, 1,717 down) DEGs (differentially expressed genes) were identitied in suc4-1 and suc4-2 compared with WT on the medium supplemented with $2 \%$ sucrose, respectively. At $4 \%$ sucrose condition, 452 (182 up, 270 down) and 1,990 (821 up, 1,169 down) DEGs were found in suc4-1 and suc4-2, respectively. Under high concentration of sucrose condition (6\% sucrose), the number of DEGs increased, which 6,485 (2,837 up, 3,648 down) and 6,354 (2,611 up, 3,743 down) DEGs were identitied in suc4-1 and suc4-2, respectively (Supplementary Fig. S6A). Besides, only 26 DEGs were overlapped in suc4-1 under these three condition, whereas 221 common DEGs were detected in suc4-2 (Supplementary Fig. S6B). KEGG enrichment analysis among the DEGs revealed that ribosome and glucosinolate biosynthesis were commonly enriched in the suc4- 1 and suc4-2 under $2 \%$ sucrose treatment, while three common enriched pathways were identified under $4 \%$ sucrose treatment (Supplementary Fig. S7A), including carbon metabolism, glyoxylate and disarboxylate metabolism and pyruvate metabolism. Additionaly, there were 12 enriched pathways overlapped in the suc4-1 and suc4-2 at 6\% sucrose condition (Supplementary Fig. S7B), which was mainly contributed by more DEGs were detected in both two mutants at this treatment than other two treatments.

\section{Atsuc4 mutants responsed to high sucrose stress through sucrose, IAA and ABA signaling pathway}

Our study show that the sucrose accumulation was much lower in atsuc4 than WT under $6 \%$ sucrose, we therefore checked the expression change of AtSUCs and AtSWEETs in atsuc4 mutants by RNA-seq analysis. As shown in Figure 7A, AtSWEET2, 14, 16 and 17 were significantly up-regulated in atsuc4 mutants compared with WT at $6 \%$ sucrose condition, whereas a reverse expression pattern were found for AtSWEET1, 4, 6, 11, 12 and 15, as well as AtSUC2. 
We further investigated the expression of AtSUC2, AtSWEET11,12 and 15 by qRT-PCR, the result showed consistence with RNA-seq data (Fig. 7B). These results revealed that more sucrose transporters tend to be down-regulated in atsuc4 mutants, which might contribute to lower level of sucrose in the mutants under high sucrose stress. Except for sucrose transport, sucrose accumulation are also affected by sucrose synthesis and degradation. Hence, we explored the expression of genes related sucrose synthesis and degradation in atsuc4 mutants under high sucrose stress. As shown in Figure 7A, three genes that encode Sucrose Synthase $(S U S 1,4,5)$ that are important for sucrose degradation, were significantly up-regulated in two mutant lines compared with WT under high sucrose stress. On the contrary, the transcript levels of the genes encoding Sucrose-Phosphate Synthase (SPS), which is involved in sucrose synthesis, were reduced significantly. Based on these results, we speculated that the pathway of sucrose degradation was active, but the sucrose synthesis was blocked by the deficiency of AtSUC4.

A significant difference in the content of ABA and IAA between atsuc4 mutants and WT under $6 \%$ sucrose stress were detected (Tab. 1). Therefore, expression of IAA and ABA-related genes were investigated by RNA-seq. As shown in Figure 7C, reduced expression was observed in two IAA biosynthesis genes (YUC2, 6), two IAA transporter genes (PIN1, 4) and five IAA signal transduction genes $(P L T 1,2, S H R, I A A 6,19)$ in two atsuc 4 mutants and WT under $6 \%$ sucrose compared with $2 \%$ sucrose treatment, while the transcript of those genes were higher in two atsuc4 mutants than WT. On the contrary, except for a negative regulator in $\mathrm{ABA}$ singal transduction $(A B / 1)$, one $\mathrm{ABA}$ biosynthesis gene (NCED3), seven ABA signal transduction genes ( $P Y L 2,8, \operatorname{SnRK2} 2.3,2.6, A B I 3,4,5)$ were up regulated in two atsuc4 mutants and WT under $6 \%$ sucrose compared with $2 \%$ sucrose treatment, while the transcript of those genes were lower in two atsuc4 mutants than WT (Fig. 7E). This rusult corresponded to the broad reduction and increased in accumulation of IAA and ABA in two atsuc4 mutants and WT under $6 \%$ sucrose treatment, respectively. But the content of IAA and ABA significantly higher and lower in suc4-1 mutants than WT, respectively. To test the reliability of transcriptome data, five sucrose metabolism genes, five IAA signaling pathway genes and five ABA signaling pathway genes were chosen for RT-qPCR assay. Gene expression as elucidated by RT-qPCR exhibited similar trends with that of the transcriptome data, with some variation in the magnitude (Fig. 7B, D, F). This result clearly suggested that deficiency of AtSUC4 had directly or indirectly stimulated the IAA signaling pathway and repressed the ABA signaling pathway under high sucrose stress.

Table 1. The WT and suc4-1 were grown on MS solid media with $2 \%$ or $6 \%$ sucrose supplementation at the 6 th day. Each sample was subjected to three biological replicates. Statistically significant differences compared with WT were determined by Duncan tests $\left({ }^{\star} P<0.05\right)$.

Table 1 The ABA and IAA contents of different plants 


\begin{tabular}{|lll|}
\hline Analyte & IAA & ABA \\
& $/\left(\mathbf{n g} \cdot \mathbf{g}^{-1} \mathrm{FW}^{-1}\right)$ & $/\left(\mathbf{n g}^{-1} \mathbf{F W}^{-1}\right)$ \\
\hline WT (2\% Suc) & $10.9 \pm 1.2$ & $6.3 \pm 0.6$ \\
\hline suc4-1 (2\% Suc) & $9.6 \pm 0.7$ & $4.1 \pm 0.2^{\star}$ \\
\hline WT (6\% Suc) & $3.2 \pm 0.3^{*}$ & $9.6 \pm 0.7$ \\
\hline suc4-1 (6\% Suc) & $4.3 \pm 0.1$ & $7.2 \pm 0.4^{*}$ \\
\hline
\end{tabular}

\section{Discussion}

As an important sucrose transporter, although various studies have focused on AtSUTs in Arabidopsis, relatively few have been performed on the effect of AtSUT4 on root development in recent years. Based on our results in this study, the possible function and the regulation mechanism of AtSUC4 in the root under high sucrose stress would be discussed.

In plants, the root as a typical sink organ that relies exclusively on an external import of sucrose for growth. Therefore, the sucrose transporter has been considered as a major determinant of plant growth for a long time [6]. Schneider et al found that no difference in growth between WT and a atsuc4 mutant in normal condition, and we confimed this result using two more atsuc4 mutants (Fig. 4E-F, Supplementary Fig. S2) [11]. In our study, we characterized a new function of AtSUC4, which it played an important role under high sucrose stress. As shown in Figure 2, with the increasing of sucrose concentration in the medium, the activity of GUS was gradually higher in pAtSUC4:: GUS plant, suggesting that AtSUC4 was strongly induced by sucrose. Thus, it is possible that the sucrose stress might be the key factor in inducing the biological function of AtSUC4. Interestingly, we found that the root length of three atsuc4 mutants were significantly longer than that of WT and AtSUC4/ suc4-1 when the sucrose concentration were $4 \%$ and $6 \%$ in the medium (Fig. 5A-B), suggesting that the deficiency of AtSUC4 enhanced the tolerance of high sucrose stress in roots, and atsuc4 mutants were less sensitive to high sucrose stress compared with WT. Besides, the sucrose content in atsuc4 mutants roots was lower compared with WT and AtSUC4/ suc4-1 under high sucrose treatment (Fig. 6A), showing the negative relationship with root length. This was consistent with the previous results [32]. However, the above difference between atsuc4 mutants and WT gradually weakened with the extension of culture time, and disappeared completely in 11 day-old seedlings, which was mainly due to photosynthesis affecting the sugar content in roots. It has been demonstrated that external application of high concentration sucrose could cause the increase of glucose content and osmotic potential on the root surrounding [33]. However, Supplementary Figure S3 and S4 showed that there was no difference in root length among all genotypes under high concentration glucose and osmotic stress (stimulated by mannitol). These data indicate that discruption of AtSUC4 plays an important role in coping with high sucrose stress in Arabidopsis, mainly by reducing the accumulation of sucrose in roots. 
Schneider et al characterized AtSUC4 in mesophyll vacuoles and found that sucrose content in AtSUC4overexpressing seedlings was reduced, indicating AtSUC4-mediated release of sucrose from the vacuolar lumen into the cytoplasm [11]. Nevertheless, we found that the deficiency of AtSUC4 lead to less sucrose accumulation in the root of atsuc4 mutants (Fig. 6A), suggesting mild conflict with the function of AtSUC4 mentioned above. In this study, AtSUC4 was localized to both plasma membrane and tonoplast by transient expression in tabacco leaves but compared to the plasma membrane labelling, the signal in tonoplast was quite faint (Supplementary Fig. S1). However, AtSUC4-Citrine gave clear and strong labelling of the plasma membrane in roots but not tonoplast by stable expression in Arabidopsis (Fig. $3 \mathrm{~A})$. Combined with the results of sucrose content in roots, we speculated that AtSUC4 may transport sucrose from extracellular into cytoplasm, resulting less sucrose accumulation in the disruption of AtSUC4.

Sucrose metabolism plays pivotal roles in development and stress response, mainly by generating metabolites to fuel growth and as signals to regulate gene expression and for crosstalk with hormonal signaling [34]. This is achieved by the generation of sugar signaling molecules or metabolic process itself [35]. Therefore, sucrose transport, synthesis and degradation are very important in regulating plant growth. To date, SUCs and SWEETs have been to characterized as the two main types of protein responsible for sucrose transport in plants [36]. AtSWEET1, 2, 4, 9-15 and 16 have been identified habour sucrose transport activity [3]. Chen et al found that sweet11;12 double mutant and sweet11;12;15 triple mutant exhibited reduced root length when germinated on sugar-free media that could be ameliorated by external sucrose addition [37]. As SWEETs facilitate sugar transport along the sugar gradient, not surprisingly, $6 \%$ sucrose treatment induced the expression of five plasma membrane localized SWEETS (AtSWEET1, 4, 11, 12, 15) in all three plants, but the transcripts of those genes were down regulated in atsuc4 mutants compared with WT. Meanwhile, the expression of two vacuolar membrane localized SWEETS (AtSWEET2, 16), which released sucrose from vocuolar lumen to cytoplasm, were shown the opposite tendency on the $6 \%$ sucrose supplementation (Fig. 7A). Unlike AtSWEETs, the function of SUCS to transport sucrose requires energy, so its expression caters to the needs of the owners. The AtSUC1 in all three plants were down-regulated with the increase of sucrose supplementation. The AtSUC2 was upregulated in WT, but down-regulated in two atsuc4s. In addition, compared with WT, the transcript level of AtSUC2 was much lower in two atsuc4s under the 6\% sucrose supplementation (Fig. 7A). We proposed that the notable decresed sucrose accumulation in the two mutants could be a result of the more downregulated expression of plasma membrane localized AtSWEETs and AtSUC2 responsible for sucrose transport than that with up-regulated expression pattern.

Sucrose metabolism plays an important role in plant growth and development [38]. On the one hand, sucrose can be hydrolysis by Invertase (INV), producing glucose and fructose. Molecular genetic studies showed that CWIN activity is required for, or even rate-limits, seed development in some instances by controlling cell division [1]. AtCWINV5 was up-regulated in three genotype plants by the stimulation of high-sucrose. Compared with WT under $6 \%$ sucrose supplementation, the expression level of AtCWINV5 in two atsuc 4 mutants were down regulated (Fig. 7A). Barratt et a1 found that reduction of CIN activity by about $40 \%$ through double mutation cinvl/cinv2, drastically reduced root growth [39]. Also, in rice, OsCyt- 
inv/ was mutated, the plant exhibited short roots [40]. When the two atsuc4 mutants and WT were planted on the medium containing $6 \%$ sucrose, the expression of AtCINV1 was down regulated. However, compared with WT, the expression of AtCINV1 was higher in the two atsuc4 mutants. On the othere hand, sucrose can be degraded by Sucrose Synthase (Sus; EC 2.4.1.13) into Hexes or their derivatives. Compared with WT under $6 \%$ sucrose supplementation, the expression level of AtSUS1, 4 and 5 in two atsuc4 mutants were up-regulated (Fig. 7A). In contrast, due to the increase of substrates, two SucrosePhosphate Synthase Enzyme (SPS; EC 2.4.1.14) genes (AtSPS1, 3) and the Sucrose Phosphatase (SPP; EC 3.1.3.24) gene (AtSPP2), which related to sucrose synthesis, were up regulated in three plant as the sucrose level increasing. While their expression level was lower in the two atsuc4s compared with WT under $6 \%$ sucrose treatment (Fig. 7). Taken together, these data suggest that AtSUC4 regulates root growth involved in many sucrose metabolic enzymes, which exert their signaling roles partly by reduce sucrose inflow and biosynthetic and increase sucrose degradation in intracellular.

Evidence from many studies highlights the central role of auxin in generation and maintenance of primary root meristems as well as orchestrating the final root architecture $[41,42]$. Thus, sucrose and auxin may operate coordinately during plant root growth. Our results showed that the roots were shorter with $6 \%$ sucrose application in both atsuc4 mutants and WT (Fig. 5), indicating that root growth was inhibited under high sucrose stress. Interestigly, the roots of atsuc4 mutants were longer than that of WT (Fig. 5). Furthermore, the IAA content in the suc4-1 root was significantly higher than that of the WT under the treatment with $6 \%$ sucrose (Tab. 1). Consistent with this, our comparative transcriptome data showed that the expression of the genes related to IAA biosynthesis (YUC2, 6) and transporters $(P I N 1,4)$ were significantly up regulated in atsuc4 mutants under high sucrose stress (Fig. 7C). The cellular levels of auxin, in turn contribute to the regulation of gene expression that defines cell fate and impacts root patterning. The PLETHORA genes are required to define the root-stem cell niche, and the transcript of these genes are strongly correlated with auxin levels [43]. This expression pattern also coincides with that of the radial patterning genes SCR and SHR, which provide parallel inputs in specifying root stem cells [44]. According to RNA-seq data, AtPLT1, 2 and AtSHR were significantly up-regulated in atsuc4 mutants compared with WT at $6 \%$ sucrose application (Fig. 7C). These results suggest that the higher concent of IAA provoked the expression of AtPLT1, 2 and AtSHR in atsuc4 mutants compared with WT, which help to keep the longer primary root under high sucrose stress. Meanwhile, the $P L T$ in turn regulate auxin accumulation in the QC both through transport (PIN4) and biosynthesis (YUCS) [45,46]. In our study, the AtPLT1 and 2 shown the same expression trend with YUC2, 6 and PIN4. Since Aux/IAA proteins are auxinsensitive repressors that have functions in the regulation of auxin-mediated root growth and development. The aux/iaa mutants, including iaa 1, 2, 13, 6, 17, 19 and 28 reduced multiple auxin responses, with smaller in size to various extents than the WT [47]. In our study, the expression of two Aux/IAAs $(I A A 6,19)$ in atsuc4 mutants were significantly higher than WT in the treatment with $6 \%$ sucrose (Fig. 7C). Taken together, our genome transcript profiling studies revealed that the deficiency of AtSUC4 declined sucrose content in atsuc4 mutants root compared with WT under $6 \%$ sucrose treatment, which could accumulated more IAA and up regulated the expression of IAA related genes, including IAA biosynthetic genes $(Y U C 2,6)$, transporters $(P I N 1,4)$, signalling trancduction $(P L T 1,2$ and $S H R)$ and 
signalling molecules $(I A A 6,19)$. Consistent with this, atsuc4 mutants had the longer root meristem zones and primary root.

ABA signaling also plays a critical role in abiotic stress and regulating root growth and root system architecture [48]. According to the data of ABA content, when the concentration of sucrose increased, the content of ABA increased significantly in both suc4-1 and WT, and it rised to a less extent in suc4-1 under $6 \%$ sucrose supplementation (Fig. 5, Tab. 1). This was in line with the situation that NCED3, a key genes that control the synthesis of ABA, was significantly decreased in two atsuc4s mutants compared with WT (Fig. 7E). The increase of ABA levels in the plant cell leads to PYR1/ PYL/ RCAR receptor-mediated inhibition of PP2C activity, which results in the activation of the SnRK2s and ultimately of the ABA signaling pathway by directly phosphorylate bZIP transcription factors ABIs $[49,50]$. In general, PP2C has a negative regulatory effect on $A B A$ signal transduction [51]. Similiarly, we found that the expression seven ABA signaling transduction genes ( $P Y L 2,8$, SnRK2.3, 2.6, $A B I 3,4,5)$ were significantly decreased in two atsuc 4 mutants with $6 \%$ sucrose supplementation, while $A B / 1$, a member of group PP2C, was significantly up regulated (Fig. 7). Yuan et al suggest that $A B I 5$ functions in the glucose-mediated inhibition of the root meristem zone by repressing PIN1 accumulation, thus leading to reduced auxin levels in roots and shorten primary root, indicating that $A B I 5 / P I N 1$ functions at the junction between $A B A$ and auxin signaling [21]. Similarly, PIN1 protein levels were reduced to a lesser extent in abi4 plants treated with ABA than in ABA-treated WT plants [52]. In our study, the reduced expression of $A B / 4,5$ and increased expression of PIN1 was found in atsuc4 mutants caompared with WT under $6 \%$ sucrose treatment. In sum, our data may indicate that the deficiency of AtSUC4 repressed the expression of $A B I 4$ and 5 in the root, which, in turn, stimulated the expression of PIN1 and accumulation of IAA content. This increased auxin activity and resulted in longer primary root.

Further physiological functional analysis of AtSUC genes are needed to explore the possible relationship between sugar signal and the functions of IAA- or ABA-related genes triggered by different stresses.

\section{Declarations}

\section{Additional Information}

\section{Consent to publish}

Not applicable.

\section{Author contributions}

S-WL, C-CP and J-ML conceived the project. S-WL, L-DZ and J-YG performed most of the experiments, TZ and T-TD performed the RNA-seq analysis. S-WL and J-ML analyzed the data and wrote the manuscript. All authors read and approved the final manuscript.

\section{Data availability}


All sequences generated in this study have been deposited in the National Center for Biotechnology Information Sequence Read Archive (https://www.ncbi.nlm.nih.gov/sra/) with project number PRJNA666009.

\section{Fundings}

This research is supported by grants from the National Natural Science Foundation of China (Grant Nos 30771759, 31170636, 31470681 and 31800560), the National Key Research and Development Program of China (Grant No.2016YFD0600104), Natural Science Foundation of Guangdong Province of China (Grant No. 2016A030311032),

Guangzhou Science and Technology Program (Grant No. 201607020024) and Foundation of Young Creative Talents in Higher Education of Guangdong Province (2017KQNCX017).

\section{Conflicts of Interest}

The authors declare that they have no competing interests.

\section{Ethics}

Not applicable.

\section{References}

1. Ruan, Y. L., Jin, Y., Yang, Y. J., Li, G. J. \& Boyer, J. S. Sugar input, metabolism and signaling mediated by invertase: roles in development, yield potential and response to drought and heat. Mol Plant. 3, 942-955 (2010).

2. Wormit, A. et al. Molecular Identification and physiological characterization of a novel monosaccharide transporter from Arabidopsis involved in vacuolar sugar transport. Plant Cell. 18, 3476-3490 (2006).

3. Braun, D. M., Wang, L. \& Ruan, Y. L. Understanding and manipulating sucrose phloem loading, unloading, metabolism and signalling to enhance crop yield and food security. Exp Bot. 65, 17131735 (2014).

4. Julius, B. T., Leach, K. A., Tran, T. M., Mertz, R. A. \& Braun, D. M. Sugar transporters in plants: new insights and discoveries. Plant Cell Physiol. 58, 1442-1460 (2017).

5. Kuhn, C. A comparison of the sucrose transporter systems of different plant species. Plant Biol. 5, 215-232 (2003).

6. Sauer, N. Molecular physiology of higher plant sucrose transporters. Febs Lett. 581, 2309-2317 (2007).

7. Eom, J. S. et al. Impaired function of the tonoplast-localized sucrose transporter in rice, OsSUT2, limits the transport of vacuolar reserve sucrose and affects plant growth. Plant Physiol. 157, 109-119 
(2011).

8. Sivitz, A. B., Reinders, A. \& Ward, J. M. Arabidopsis sucrose transporter AtSUC1 is important for pollen germination and sucrose-induced anthocyanin accumulation. Plant Physiol. 147, 92-100 (2008).

9. Gottwald, J. R., Krysan, P. J., Young, J. C., Evert, R. F. \& Sussman, M. R. Genetic evidence for the in planta role of phloem-specific plasma membrane sucrose transporters. Proc Natl Acad Sci U S A. 97, 13979-13984 (2000).

10. Endler, A. et al. Identification of a vacuolar sucrose transporter in barley and Arabidopsis mesophyll cells by a tonoplast proteomic approach. Plant Physiol. 141, 196-207 (2006).

11. Schneider, S. et al. Vacuoles release sucrose via tonoplast-localised SUC4-type transporters. Plant Biol (Stuttg). 14, 325-336 (2012).

12. Freixes, S., Thibaud, M.C., Tardieu, F. \& Muller, B. Root elongation and branching is related to local hexose concentration in Arabidopsis thaliana Plant Cell Environ. 25, 1357-1366 (2002).

13. Kircher, S. \& Schopfer, P. Photosynthetic sucrose acts as cotyledon-derived long-distance signal to control root growth during early seedling development in Arabidopsis. Proc Natl Acad Sci U S A. 109, 11217-11221 (2012).

14. Durand, M. et al. Carbon source-sink relationship in Arabidopsis thaliana: the role of sucrose transporters. Planta. 247, 587-611 (2018).

15. Hoth, S., Niedermeier, M., Feuerstein, A., Hornig, J. \& Sauer, N. An ABA-responsive element in the AtSUC1 promoter is involved in the regulation of AtSUC1 expression. Planta. 232, 911-923 (2010).

16. Chaiwanon, J., Wang, W., Zhu, J. Y., Oh, E. \& Wang, Z. Y. Information integration and vommunication in plant growth regulation. Cell. 164, 1257-1268 (2016).

17. Macgregor, D. R., Deak, K. I., Ingram, P. A. \& Malamy, J. E. Root system architecture in Arabidopsis grown in culture is regulated by sucrose uptake in the aerial tissues. Plant Cell. 20, 2643-2660 (2008).

18. Zhang, S., Peng, F., Xiao, Y., Wang, W. \& Wu, X. Peach PpSnRK1 participates in sucrose-mediated root growth through auxin signaling. Front Plant Sci. 11, 409 (2020).

19. Antoni, R. et al. PYRABACTIN RESISTANCE1-LIKE8 plays an important role for the regulation of abscisic acid signaling in root. Plant Physiol. 161, 931-941 (2013).

20. Yu, B. et al. Genome-wide binding analysis reveals that $A N A C O 60$ directly represses sugar-induced transcription of ABI5 in Arabidopsis. Plant J. 103, 965-979 (2020).

21. Yuan, T. T., Xu, H. H., Zhang, K. X., Guo, T. T. \& Lu, Y. T. Glucose inhibits root meristem growth via $A B A$ INSENSITIVE 5, which represses PIN1 accumulation and auxin activity in Arabidopsis. Plant Cell Environ. 37, 1338-1350 (2014).

22. Clough, S. J. \& Bent, A. F. Floral Dip: A simplified method for Agrobacterium-mediated transformation of Arabidopsis thaliana. Plant J. 16, 735-743 (1998).

23. $\mathrm{Ma}, \mathrm{X}$. et al. A robust CRISPR/Cas9 system for convenient, high-efficiency multiplex genome editing in monocot and dicot plants. Mol Plant. 8, 1274-1284 (2015). 
24. Jiang, S. Y., Vanitha, J., Bai, Y. \& Ramachandran, S. A novel binary T-vector with the GFP reporter gene for promoter characterization. Plos One. 9, e107328 (2014).

25. Erwig, J. et al. Chitin-induced and CHITIN ELICITOR RECEPTOR KINASE1 (CERK1) phosphorylationdependent endocytosis of Arabidopsis thaliana LYSIN MOTIF-CONTAINING RECEPTOR-LIKE KINASE5 (LYK5). New Phytol. 215, 382-396 (2017).

26. Batistic, O., Waadt, R., Steinhorst, L., Held, K. \& Kudla, J. CBL-mediated targeting of CIPKs facilitates the decoding of calcium signals emanating from distinct cellular stores. Plant J. 61, 211-222 (2010).

27. Robert, S., Zouhar, J., Carter, C. \& Raikhel, N. Isolation of intact vacuoles from Arabidopsis rosette leaf-derived protoplasts. Nat Protoc. 2, 259-262 (2007).

28. Fan, R. C. et al. Apple sucrose transporter SUT1 and sorbitol transporter SOT6 interact with cytochrome b5 to regulate their affinity for substrate sugars. Plant Physiol. 150, 1880-1901 (2009).

29. Karimi, M., Inze, D. \& Depicker, GATEWAY ${ }^{\mathrm{TM}}$ vectors for Agrobacterium-mediated plant transformation. Trends Plant Sci. 7, 193-195 (2002).

30. Schneider, S., Beyhl, D., Hedrich, R. \& Sauer, N. Functional and physiological characterization of Arabidopsis INOSITOL TRANSPORTER1, a novel tonoplast-localized transporter for myo-inositol. Plant Cell. 20, 1073-1087 (2008).

31. Chen, M. L. et al. Highly sensitive and quantitative profiling of acidic phytohormones using derivatization approach coupled with nano-LC-ESI-Q-TOF-MS analysis. J Chromatogr B Analyt Technol Biomed Life Sci. 905, 67-74 (2012).

32. Gong, X.et al. Arabidopsis AtSUC2 and AtSUC4, encoding sucrose transporters, are required for abiotic stress tolerance in an ABA-dependent pathway. Physiol Plant. 153, 119-136 (2015).

33. Veillet, F., Gaillard, C., Coutos-Thevenot, P. \& La Camera, S. Targeting the AtCWIN1 gene to explore the role of invertases in sucrose transport in roots and during botrytis cinerea infection. Front Plant Sci. 7, 1899 (2016).

34. Liang, Y. et al. Auxin regulates sucrose transport to repress petal abscission in rose (Rosa hybrida). Plant Cell. 32, 3485-3499 (2020).

35. O'Hara, L. E., Paul, M. J. \& Wingler, A. How do sugars regulate plant growth and development? New insight into the role of trehalose-6-phosphate. Mol Plant. 6, 261-274 (2013).

36. Chen, L. Q., Cheung, L. S., Feng, L., Tanner, W. \& Frommer, W. B. Transport of sugars. Annu Rev Biochem. 84, 865-894 (2015).

37. Chen, L. Q. et al. A cascade of sequentially expressed sucrose transporters in the seed coat and endosperm provides nutrition for the Arabidopsis embryo. Plant Cell. 27, 607-619 (2015).

38. Ruan, Y. L. Sucrose metabolism: Gateway to diverse carbon use and sugar signaling. Annu Rev Plant Biol. 65, 33-67 (2014).

39. Barratt, D.H.P.et al. Normal growth of Arabidopsis requires cytosolic invertase but not sucrose synthase. Proc Natl Acad Sci U S A. 106, 13124 (2009). 
40. Jia, L. et al. OsCYT-INV1 for alkaline/neutral invertase is involved in root cell development and reproductivity in rice (Oryza sativa L.). Planta. 228, 51-59 (2008).

41. Dello, I. R. et al. A genetic framework for the control of cell division and differentiation in the root meristem. Science. 322, 1380-1384 (2008).

42. Overvoorde, P., Fukaki, H. \& Beeckman, T. Auxin control of root development. Cold Spring Harb Perspect Biol. 2, a1537 (2010).

43. Aida, M. et al. The PLETHORA genes mediate patterning of the Arabidopsis root stem cell niche. Cell. 119, 109-120 (2004).

44. Moreno-Risueno, M. A. et al. Transcriptional control of tissue formation throughout root development. Science. 350, 426-430 (2015).

45. Galinha, C. et al. PLETHORA proteins as dose-dependent master regulators of Arabidopsis root development. Nature. 449, 1053-1057 (2007).

46. Santuari, L. et al. The PLETHORA gene regulatory network guides growth and cell differentiation in Arabidopsis Plant Cell. 28, 2937-2951 (2016).

47. Mockaitis, K. \& Estelle, M. Auxin receptors and plant development: A new signaling paradigm. Annu Rev Cell Dev Biol. 24, 55-80 (2008).

48. Gong, Z. et al. Plant abiotic stress response and nutrient use efficiency. Sci China Life Sci. 63, 635674 (2020).

49. Ma, Y. et al. Regulators of PP2C phosphatase activity function as abscisic acid sensors. Science. 324, 1064-1068 (2009).

50. Park, S. Y. et al. Abscisic acid inhibits type $2 \mathrm{C}$ protein phosphatases via the PYR/PYL family of START proteins. Science. 324, 1068-1071 (2009).

51. Schweighofer, A., Hirt, H. \& Meskiene, I. Plant PP2C phosphatases: Emerging functions in stress signaling. Trends Plant Sci. 9, 236-243 (2004).

52. Shkolnik-Inbar, D. \& Bar-Zvi, D. AB/4 mediates abscisic acid and cytokinin inhibition of lateral root formation by reducing polar auxin transport in Arabidopsis. Plant Cell. 22, 3560-3573 (2010).

\section{Figures}




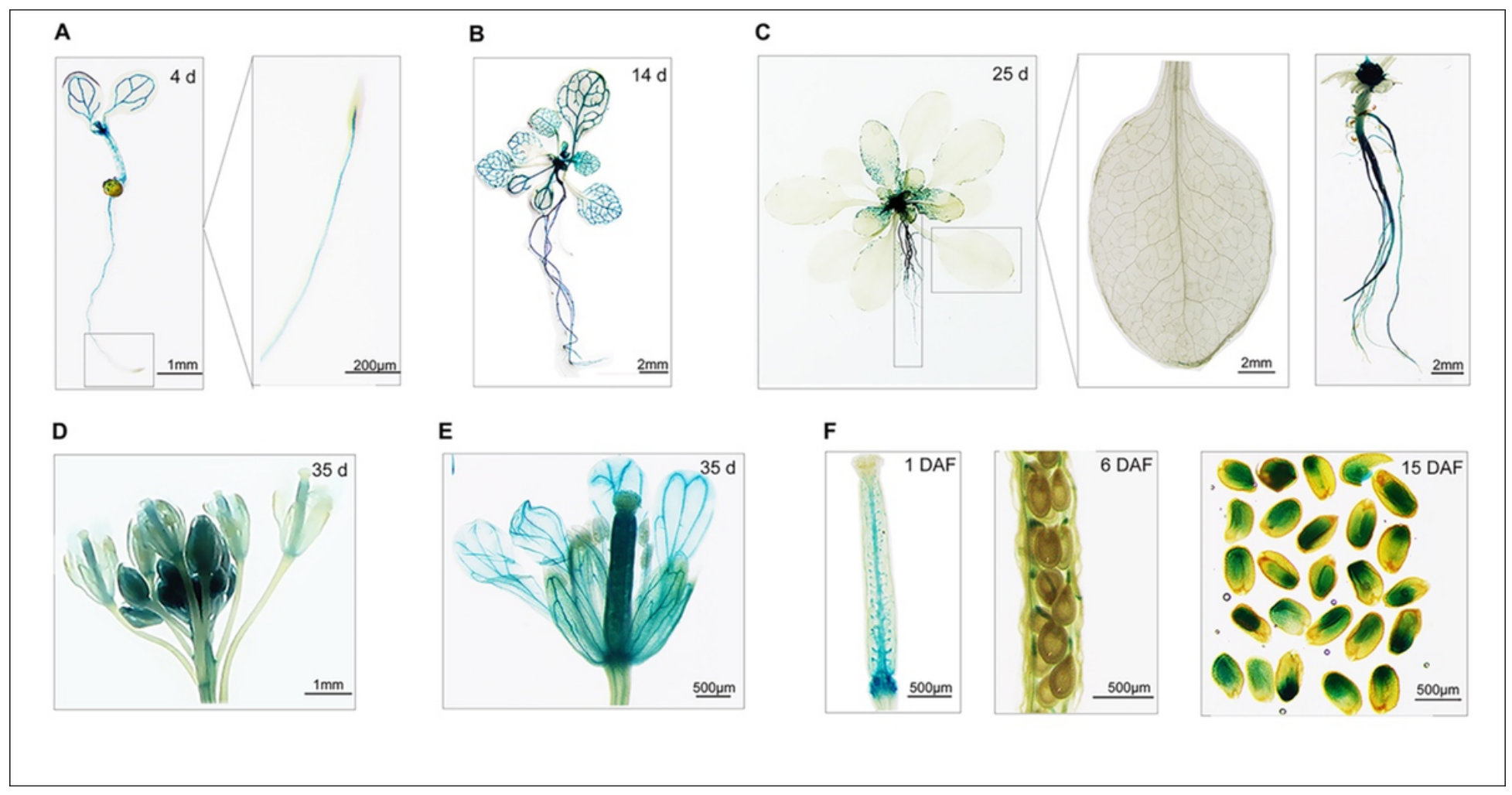

Figure 1

Expression pattern of the AtSUC4 in life cycle of Arabidopsis by pAtSUC4::GUS expression. (A) Seedlings grown for 4 days on MS solid media with 1\% sucrose supplementation. (B) Seedlings grown for 14 days on MS solid media with $1 \%$ sucrose supplementation. (C) The rosette leaves and root of the whole plant grown for 25 days in soil. (D) The inflorescence for 35 days in soil. (E) The stigma and vascular tissue of petals for 35 days in soil. (F) The siliques of Arabidopsis at 1, 6 and 15 DAF (days after flowering). 
A
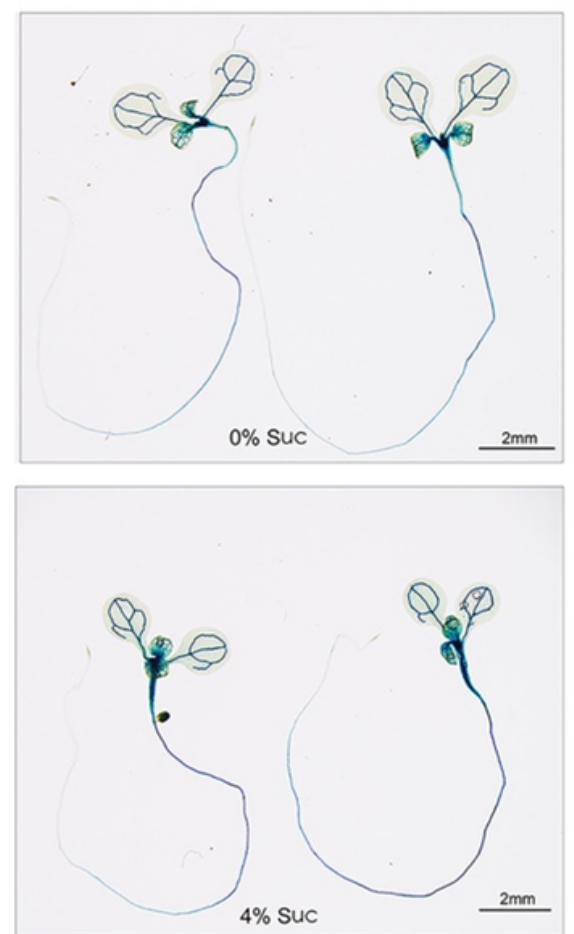
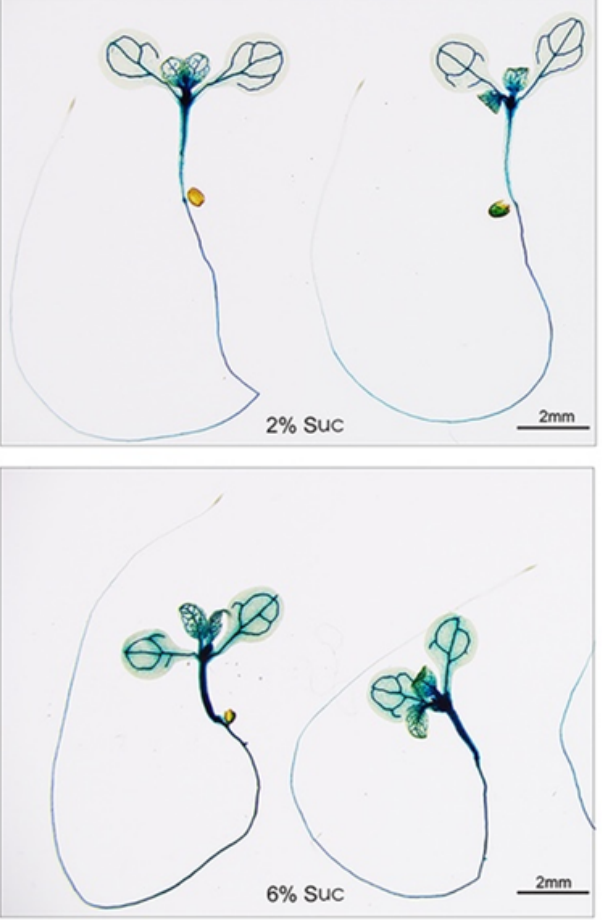

B

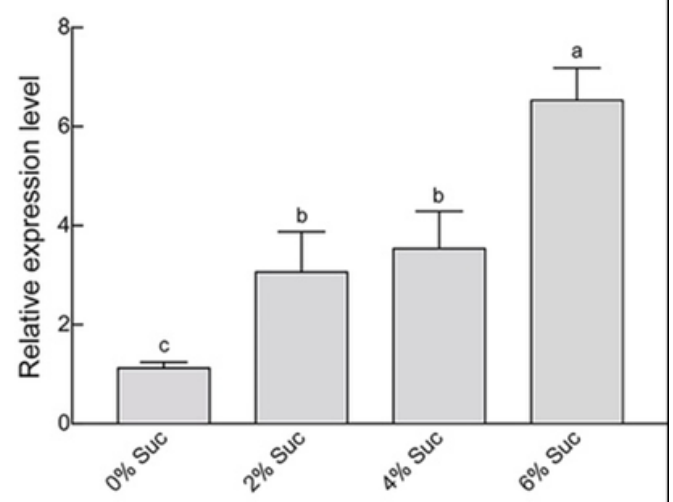

\section{Figure 2}

Expression level of AtSUC4 in response to different concentrations of sucrose. (A) 7 d-old pAtSUC4::GUS seedling on MS solid media with different concentrations of sucrose $(0 \%, 2 \%, 4 \%$ and $6 \%)$. (B) The relative expression level of GUS in roots of pAtSUC4::GUS plants. Values represent the mean \pm SE of three biological replicates. Bars with the different letters indicate a significantly difference among treatments for a same stage according to Duncan test (at $\mathrm{P}<0.05$ ).
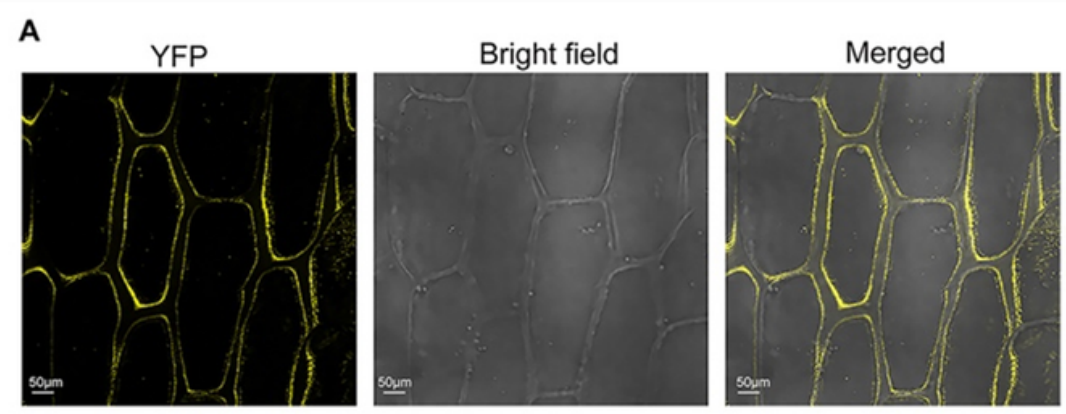

\section{B}
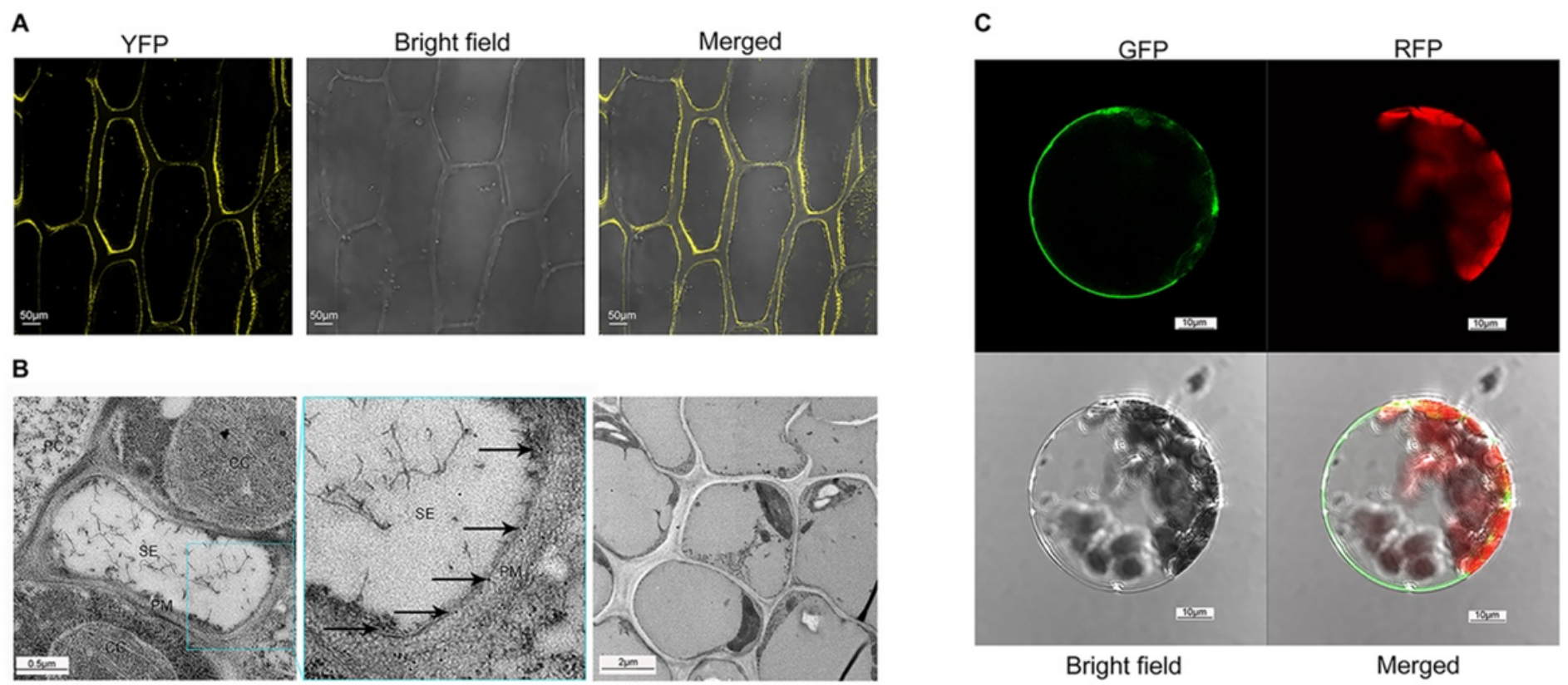

Bright field

Merged 


\section{Figure 3}

The subcellular localization of AtSUC4. (A) The root imaged of Citrine fusion to the C-terminus of AtSUT4 by stably transformed. (B) The imaged by immune colloidal gold. SE: sieve element, CC: companion cell, PM: plasma membrane; The black arrow represents the gold particles. The right image served as a negative control. (C) The subcellular localization of a GFP fusion to the C-terminus of AtSUT4 by transient transformation of Arabidopsis protoplasts.

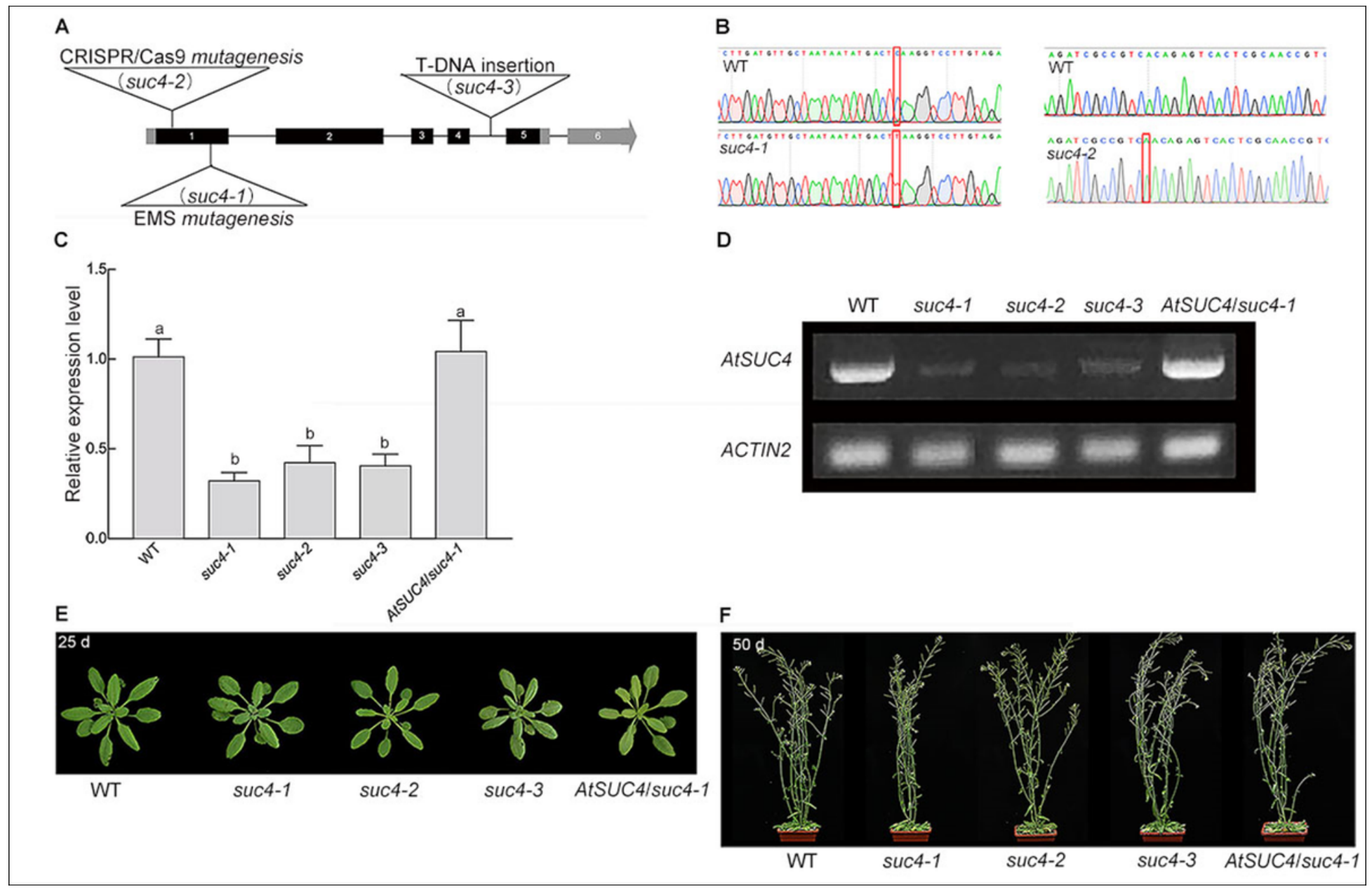

\section{Figure 4}

Phenotypic characterizations of atsuc4 mutants and complement-expressor (AtSUC4/suc4-1) plants. (A) Schematic diagram of the mutagenesis types of three atsuc4 mutants and their mutant sites. suc4-1 from ethyl-methanesulfonate (EMS) mutagenesis, suc4-2 from CRISPR/Cas9 mutagenesis and suc4-3 from T-DNA insertion mutagenesis. (B) Comparison of sequencing results of mutant suc4-1 and suc4-2 compared with WT. (C) and (D) Reverse transcription-quantitative and semi-quantitative real time PCR (RT-qPCR) expression analyses of AtSUC4 transcript in three mutants and the complement-expressor line. The phenotye of $25 \mathrm{~d}$-old rosettes (E) and $50 \mathrm{~d}$-old plants (F) from WT, atsuc4 mutants and complementexpressor plant shown no difference. Values in (C) represent the mean \pm SE of three biological replicates. Bars with the different letters indicate a significantly difference among different plants for a same stage according to Duncan test (at $P<0.05)$. 


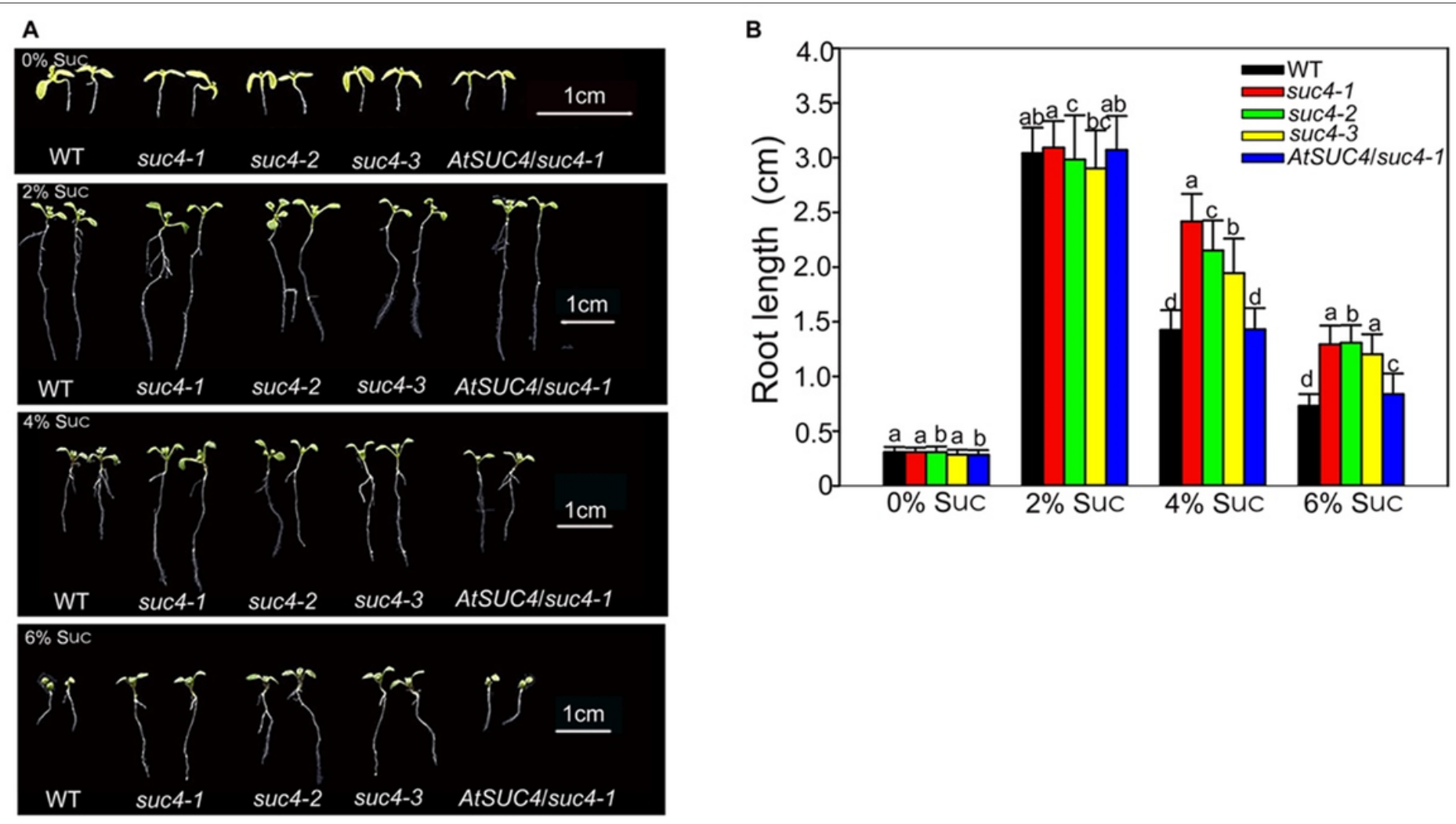

\section{Figure 5}

Atsuc4 mutants reduced the inhibition of high concentration sucrose stress on root growth at 6 days after sowing. (A) The root phenotype of different lines under different concentrations of sucrose treatment $(0 \%$, $2 \%, 4 \%$ and $6 \%$ ). (B) The statistics of root length among various concentrations of sucrose treatment.

Values in (B) represent the mean \pm SE of three biological replicates. Bars with the different letters indicate a significantly difference among different genotypes for the same sucrose concentration treatment according to Duncan test (at $P<0.05), n>50$.

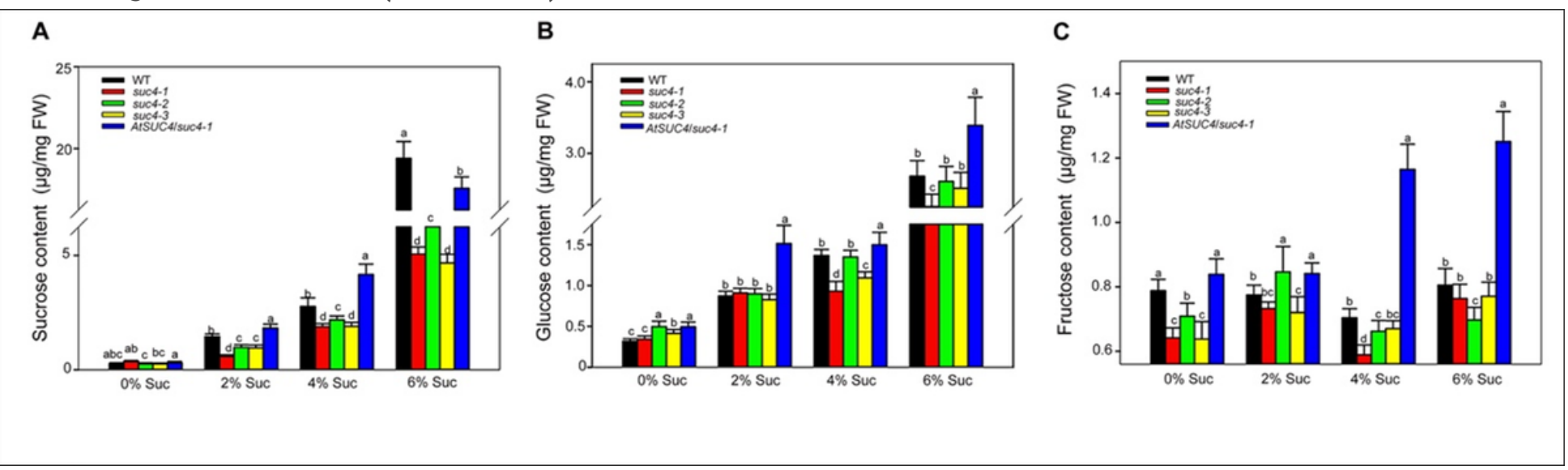

Figure 6 
The concentration of different sugars (including sucrose, glucose and fructose) in roots from all genotypes which were cultivated on MS solid media with different concentration of sucrose $(0 \%, 2 \%, 4 \%$ and 6\%) at the 6th day. (A) Sucrose content. (B) Glucose content. (C) Fructose content. Values in (A), (B) and $(C)$ represent the mean $\pm S E$ of three biological replicates. Bars with the different letters indicate a significantly difference among different genotypes for the same sucrose concentration treatment according to Duncan test (at $\mathrm{P}<0.05$ ), $\mathrm{n}>30$.

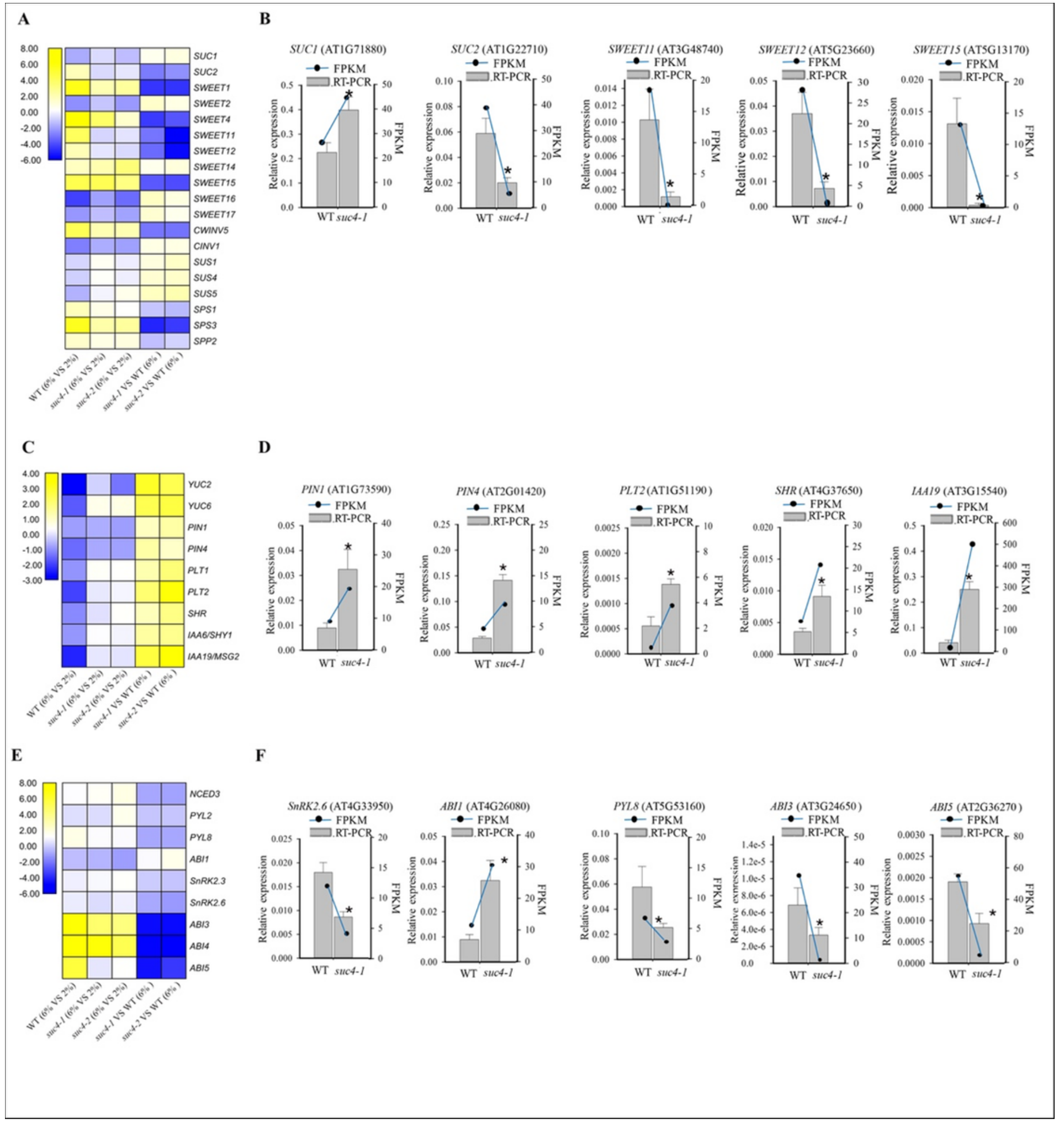


The expression level of genes related to sucrose, IAA and ABA signaling pathwa signaling pathway in root of different plants which were grown on MS solid media with $2 \%, 4 \%$ or $6 \%$ sucrose for 6 days. Each sample was subjected to three biological replicates. $(A)(C)(E)$ Heatmap of representative genes expression related to sucrose, IAA and ABA signaling pathway. (B)(D)(F) Gene expression changes as assessed by RT-qPCR. The relative expression detected by RT-qPCR, was represented by a bar graph, and the left ordinate indicated the expression level. FPKM of genes, according to RNA-Seq data, was represented by a line graph, and the right ordinate indicated the FPKM level. The asterisk represented a significant difference compared with WT. The log2 fold change value of the gene expressions from transcriptome is displayed in different colors. Yellow color means high expression and blue color means low expression.

\section{Supplementary Files}

This is a list of supplementary files associated with this preprint. Click to download.

- SupplementaryDataS1.xlsx

- SupplementaryinformationSR.pdf 